\title{
CRÓNICA DE LA LEY 27811: 16 AÑOS DESPUÉS, ACIERTOS Y DESAFÍOS EN LA PROTECCIÓN DE LOS CONOCIMIENTOS TRADICIONALES DE LOS PUEBLOS INDÍGENAS ASOCIADOS A LA DIVERSIDAD BIOLÓGICA.
}

\author{
CHRONICLE OF THE LAW 27811: 16 YEARS AFTER, STRENGTHS AND \\ CHALLENGES IN THE PROTECTION OF TRADITIONAL KNOWLEDGE OF \\ INDIGENOUS PEOPLES DERIVED FROM BIOLOGICAL RESOURCES.
}

\author{
Joyce Lenna Ticona Núñez ${ }^{1}$ \\ "El conocimiento es poder" \\ Francis Bacon
}

\section{Resumen:}

El artículo describe el proceso de elaboración y aprobación de la Ley $\mathrm{N}^{\circ}$ 27811, Ley que establece el Régimen de Protección de los Conocimientos Colectivos de los Pueblos Indígenas vinculados a los Recursos Biológicos; y analiza brevemente algunas disposiciones que se vienen aplicando y que en consecuencia constituyen un avance práctico en la protección de los conocimientos tradicionales de los pueblos indígenas; igualmente, reflexiona sobre las posibles razones por las que algunos delos principales componentes de la Ley, como la suscripción de contratos de licencia o el Fondo para el Desarrollo de los Pueblos Indígenas, aún no han sido materia de implementación.

Palabras claves: conocimiento colectivo, conocimiento tradicional, consentimiento informado previo, contratos de licencia, fondo para el desarrollo de los pueblos indígenas, ley 27811, propiedad intelectual, pueblos indígenas, registros.

\section{Abstract:}

The article describes the process of developing and enacting the Law $N^{\circ} 27811$, Law introducing a protection regime for the collective knowledge of indigenous peoples derived from biological resources; and briefly analyze some provisions

Abogada y Magister en Derecho de la Propiedad Industrial por la Universidad Panthéon Assas - Paris 2 y Magister en Derecho Ambiental y Desarrollo Sostenible por la Universidad Panthéon Sorbona - Paris 1. Consultora en acceso a recursos genéticos, conocimientos tradicionales, variedades vegetales, semillas, bioseguridad, gestión de biodiversidad, transferencia de tecnología y propiedad intelectual. Correo electrónico: lennaticona@gmail.com 
successfully applied which therefore constitute a practical advance in the protection of traditional knowledge of indigenous peoples; also ponder about the possible causes for the absence of license contracts or the implementation of the Fund for the Development of Indigenous Peoples, as main themes of the Law.

Key words: collective knowledge, traditional knowledge, prior informed consent, intellectual property, license contracts, fund for the development of indigenous peoples, law 27811, indigenous peoples, registers.

\section{INTRODUCCIÓN}

A finales de 1995, y en gran medida por las negociaciones relacionadas al Programa Internacional de Cooperación para la Biodiversidad (ICBG) (Tobin B y Taylor E., 2009, p.41), germinaban las primeras reflexiones sobre cómo plantear un régimen nacional de protección de conocimientos tradicionales ${ }^{2}$. Este contexto propició que a inicios de 1996, el Instituto Nacional de Defensa de Competencia y de la Propiedad Intelectual-INDECOPI y el entonces denominado Ministerio de Agricultura, conformaran cinco grupos de trabajo con el objetivo de elaborar la propuesta de marco legal para la protección de los conocimientos de las comunidades indígenas.

Coadyuvando a esta iniciativa, la entonces Ley de Propiedad Industrial aprobada mediante Decreto Legislativo $\mathrm{N}^{\circ} 823$ y publicada en el Diario Oficial El Peruano el 24 de abril de 199633, incluyó disposiciones para el establecimiento de un régimen de protección y, de ser el caso, un registro, de los conocimientos de las comunidades nativas y campesinas ${ }^{4}$.

El grupo de trabajo encargado de elaborar una propuesta de marco legal para la protección de los conocimientos tradicionales de las comunidades indígenas elaboró un primer y segundo borrador en junio de 1996 y agosto de 1997, respectivamente. El documento fue circulado entre expertos del tema para finalmente lograr una propuesta mejorada en abril de 1999. Por otro lado, dado el interés de validar la propuesta con los propios poseedores del conocimiento tradicional y bajo el sustento legal del Convenio $\mathrm{N}^{\circ} 169$ de

Reunión de Trabajo entre SPDA y el INDECOPI. En: RUIZ, Manuel. La protección jurídica de los conocimientos tradicionales: Algunos Avances Políticos y Normativos en América Latina. Lima: Unión Mundial para la Naturaleza/ Sociedad Peruana de Derecho Ambiental. 2006, p. 128

El Decreto Legislativo No 823 estuvo vigente de mayo de 1996 a febrero de 2009 fecha que entró en vigor el Decreto Legislativo № 1075, Decreto Legislativo que aprueba Disposiciones Complementarias a la Decisión 486 de la Comunidad Andina que establece el Régimen Común sobre Propiedad Industrial.

$4 \quad$ El artículo 63 del Decreto Legislativo $\mathbf{N}^{\circ} 823$ señalaba que por Decreto Supremo refrendado por el Ministerio de Industria, Turismo, Integración y Negociaciones Comerciales Internacionales, podrá establecerse un régimen especial de protección y, de ser el caso, un registro, de los conocimientos de las comunidades nativas y campesinas. 
la $\mathrm{OIT}^{5}$, se presentó la propuesta en dos talleres dirigidos a representantes de los pueblos indígenas durante abril y mayo de 19996. Igualmente se realizó una presentación en el Seminario Internacional "Conocimientos Tradicionales y Recursos Genéticos: Una oportunidad en el mercado global" co-organizado por el INDECOPI y la Organización Mundial de la Propiedad Intelectual, del 19 al 21 de mayo de 1999.

Producto de este trabajo organizado y multisectorial, liderado por el INDECOPI y los entonces denominados Ministerio de Promoción de la Mujer y del Desarrollo Humano, Ministerio de Agricultura y Ministerio de Pesquería7; el 21 de octubre de 1999 se publicó en Separata Especial del Diario Oficial El Peruano, el Documento de Trabajo N 003-1999 titulado "Propuesta de Régimen de Protección de los Conocimientos Colectivos de los Pueblos Indígenas y Acceso a los Recursos Genéticos", el cual contenía el estudio y análisis de la realidad de ambas materias en el Perú, así como las propuestas normativas de las mismas.

El proceso de difusión de este documento comprendió además de su publicación a fines de recibir comentarios, reuniones con gremios ${ }^{8}$, una presentación ante la Comisión de Ambiente, Ecología y Amazonía del Congreso de la República y varios talleres y mesas redondas posteriores ${ }^{9}$.

Extendiéndose el plazo para la recepción de comentarios ${ }^{10}$ hasta el 22 de mayo de 2000 se recibieron más de treinta aportes de diferentes sectores e instituciones nacionales e internacionales, que dieron como resultado la propuesta normativa contemplada en el Anexo B del Documento de Trabajo N 010-2000. Área de Estudios Económicos del INDECOPI. "Propuesta de régimen de protección de los conocimientos colectivos de los Pueblos Indígenas (y algunas reflexiones sobre la regulación del acceso a los recursos genéticos)" de

Artículo 6 inciso a) del Convenio 169 que dispone que se debe realizar una consulta a los pueblos indígenas cada vez que prevean medidas legislativas o administrativas susceptibles de afectarles directamente.

6 El primer taller se realizó en Lima los días 26 y 27 de abril de 1999 el mismo que contó con la participación de 16 representantes nacionales de pueblos indígenas; el segundo taller se llevó a cabo en Urubamba, Cusco los días 10,11 y 12 de mayo de 1999 donde participaron 36 representantes de los pueblos indígenas.

7 El mismo que además conto con la participación de varias instituciones en diferentes etapas, teniendo un rol protagónico: Asociación Interétnica de Desarrollo de la Selva Peruana (AIDESEP), Consejo Nacional del Ambiente (CONAM), Confederación de Nacionalidades Amazónicas del Perú (CONAP), Centro de Estudios y Promoción del Desarrollo (DESCO), Instituto del Mar del Perú (IMARPE), INDECOPI, Instituto Nacional de Investigación Agraria (INIA), Instituto Nacional de Recursos Naturales (INRENA), Instituto Indigenista Peruano, Ministerio de Industria, Turismo, Integración y Negociaciones Internacionales (MITINCI), SPDA, Universidad Agraria La Molina.

8 Cámara de Comercio Internacional, CONFIEP, SECREX - Sociedad de Comercio Exterior del Perú, Sociedad Nacional de Industrias, así como con la Asociación de Industrias Farmacéuticas de Origen y Capital Nacionales (ADIFAN) y con la Asociación Nacional de Laboratorios Farmacéuticos (ALAFARPE).

9 Detalladas en el Documento de Trabajo N 010-2000. Área de Estudios Económicos del INDECOPI. “Propuesta de régimen de protección de los conocimientos colectivos de los Pueblos Indígenas (y algunas reflexiones sobre la regulación del acceso a los recursos genéticos). Preparado por Juan Luis Avendaño Cisneros, Ana María Pacón, Gonzalo Ruiz y Begoña Venero, con la colaboración de Sylvia Bazán, Armando Cáceres y Beatriz Boza. Agosto, 2000.

10 Periodo de recepción de comentarios fue del 21 de octubre de 1999 al 20 de diciembre de 1999 inicialmente, el cual fue ampliado al 21 de febrero de 2000 y nuevamente al 22 de mayo de 2000. 
fecha agosto de 2000. Sin embargo, del total de aportes recibidos, solo dos pertenecían a representantes de los pueblos indígenas lo cual evidenciaba el fracaso en conseguir involucrar efectivamente a los poseedores de los conocimientos tradicionales en el proceso participativo de la elaboración de la propuesta normativa (Tobin B. 2001, p. 53) ; así y en tanto las organizaciones indígenas manifestaron su desacuerdo con el contenido de la propuesta normativa, el INDECOPI decidió no presentar el borrador de la propuesta ante el Congreso (Tobin y Swiderska K., 2001, p.10) bajo el fundamento de la necesidad de promover un nuevo proceso y mayores consultas.

Sin embargo, a pesar de este estancamiento, las reuniones, talleres y los debates que se originaron durante el proceso de elaboración de la propuesta normativa evidenciaron la urgente necesidad de contar con mecanismos para la protección del conocimiento tradicional. En respuesta a este escenario, en el año 2002 se presentaron los siguientes proyectos de ley, todos con el objetivo común de establecer el régimen especial de protección de los conocimientos colectivos de los pueblos indígenas: Proyecto de Ley N²754/2001-CR, Ley que protege la propiedad intelectual comunitaria: comunidades campesinas y nativas, presentado por el congresista Yohny Lescano Ancieta con fecha 6 de mayo de 2002; Proyecto de Ley 2439/2001-CR, Ley que establece un Régimen de Protección de los Conocimientos Colectivos de los Pueblos Indígenas, presentado por el congresista Héctor Chávez Chuchón con fecha 20 de mayo de 2002; y el Proyecto de Ley 3275/2001-CR, Ley que crea el Régimen de Protección de los Conocimientos Colectivos de los Pueblos y Comunidades Indígenas vinculados a los recursos biológicos, presentado por el Grupo Parlamentario Perú Posible con fecha 27 de junio de 2002.

El Dictamen de la Comisión de Asuntos Indígenas y Afroperuanos del Congreso de la República de fecha 09 de julio de 2002, recaído en los mencionados proyectos señaló a las iniciativas legislativas como un precedente en el quehacer jurídico nacional en tanto pretendían establecer el reconocimiento del Estado Peruano del derecho y la facultad de los Pueblos Indígenas de decidir sobre sus conocimientos colectivos, con el objetivo común de establecer un régimen especial de protección y concluyó recomendando la aprobación de los mismos con un texto sustitutorio. En la 13ava Sesión de la Comisión Permanente del Congreso de la República, de fecha 18 de julio de 2002, el texto sustitutorio de 71 artículos, 2 disposiciones complementarias, una transitoria y una final fue aprobado por 18 votos a favor, ninguno en contra y ninguna abstención.

Finalmente, el 10 de agosto de 2002 fue publicada en el Diario Oficial El Peruano, la Ley $N^{\circ}$ 27811, Ley que establece el Régimen de Protección 
de los Conocimientos Colectivos de los Pueblos Indígenas vinculados a los Recursos Biológicos.

No obstante lo expuesto, y a fines del presente artículo quisiéramos mencionar algunos elementos de interés sobre los proyectos de Ley antes mencionados.

En relación al Proyecto de Ley $N^{\circ} 2754 / 2001-C R$, Ley que protege la propiedad intelectual comunitaria: comunidades campesinas y nativas, mencionar que su ámbito establecía la protección intelectual comunitaria aplicada a los conocimientos tradicionales, creaciones, diseños, patrimonio cultural, artesanía, alfarería, música, gastronomía, danza , arte y otros de todos los grupos étnicos culturales que tengan derechos intelectuales comunitarios.

Igualmente, se hace notar el planteamiento del Proyecto de Ley $\mathrm{N}^{\circ}$ 2934/2001-CR el cual recogía íntegramente la propuesta elaborada por el INDECOPI, donde se proponían aspectos como definiciones de comunidades campesinas y nativas; y en especial: 1) La mención del mínimo del $0.5 \%$ del valor de las ventas brutas antes de impuestos resultantes de la comercialización de los productos desarrollados a partir de dicho conocimiento colectivo al Fondo para el Desarrollo de los Pueblos Indígenas; 2) La mención de regalía del mínimo del $0.5 \%$ del valor de las ventas brutas antes de impuestos resultantes de la comercialización de los productos desarrollados a partir de los conocimientos colectivos que se encuentre en el dominio público destinado para el Fondo; y 3) Dentro de los requisitos del contrato de licencia el establecimiento de regalías que incluían "un pago inicial o alguna forma de compensación directa e inmediata a los pueblos indígenas y un porcentaje (no definido) del valor de la venta bruta antes de impuestos resultantes de la comercialización de los productos desarrollados directa e indirectamente a partir de dicho conocimiento colectivo"11.

Sin embargo, fue del Proyecto de Ley 3275/2001-CR que los legisladores recogieron la mayor cantidad de propuestas de artículos que hoy se aprecian en la actual Ley 27811 y donde se hace referencia a: 1) La precisión del porcentaje del 10\% del valor de las ventas brutas antes de impuestos resultantes de la comercialización de los productos desarrollados a partir de dicho conocimiento colectivo al Fondo para el Desarrollo de los Pueblos Indígenas; 2) Al porcentaje no definido cuando se trate de uso de conocimientos tradicionales que se encuentren en el dominio público para el Fondo y; 3) Al establecimiento de compensaciones en el contrato de licencia que incluyen un pago inicial monetario $u$ otro equivalente dirigido

\footnotetext{
11 Proyecto de Ley $N^{\circ}$ 2439/2001-CR, artículo 24, Contenido del contrato de licencia.
} 
a su desarrollo sostenible; y al porcentaje no menor del $5 \%$ del valor de las ventas brutas antes de impuestos resultantes de la comercialización de los productos desarrollados directa e indirectamente a partir del conocimiento colectivo objeto del contrato de licencia.

El presente artículo consta de la siguiente estructura: i) Parte Introductoria donde se plasma la crónica del proceso de elaboración y aprobación de la Ley 27811; ii) Una primera parte donde se exponen lo que se considera algunos de los aciertos y logros de la Ley $\mathrm{N}^{\circ} 27811$; iii) Una segunda parte donde se analizan algunos de los elementos que por la complejidad de su puesta en práctica, constituyen los desafíos actuales en la implementación de la Ley $\mathrm{N}^{\circ} 27811$ y iv) Parte Final donde se exponen algunas consideraciones y recomendaciones en un deseo de contribuir en las reflexiones sobre la implementación de la Ley $\mathrm{N}^{\circ} 27811$.

\section{PRIMERA PARTE: LOS ACIERTOS}

\section{RECONOCIMIENTO DE DERECHOS}

El reconocimiento del Estado peruano sobre el derecho y facultad de los pueblos y comunidades indígenas sobre sus conocimientos colectivos se desprende de la Constitución Política del Perú ${ }^{12}$; de las disposiciones del artículo 8j del Convenio sobre la Diversidad Biológica ${ }^{13}$; del artículo 7 de la Decisión 391 de la CAN ${ }^{14}$; y los artículos 23 y 24 de la Ley de Conservación y Uso Sostenible de la Diversidad Biológica ${ }^{15}$.

Se hace notar que el derecho de los Pueblos Indígenas sobre sus conocimientos tradicionales nace por el simple hecho de poseerlos, en reconocimiento que los mismos forman parte de su identidad cultural y étnica ${ }^{16}$, de su contribución al desarrollo o a la preservación de estos.

Los derechos atribuidos son inalienables en tanto los conocimientos tradicionales son un legado de las generaciones pasadas para las presentes y futuras. En el mismo sentido, estos derechos son imprescriptibles forman parte del Patrimonio Cultural de los Pueblos Indígenas ${ }^{17}$.

\footnotetext{
12 Artículo 2, inciso 19, de la Constitución Política del Perú.

13 El mismo que establece la obligación de los Estados de respetar, preservar y mantener los conocimientos, innovaciones y prácticas de las comunidades indígenas y locales.

14 El cual reconoce, sujeto a la legislación nacional, el derecho de las comunidades indígenas sobre sus conocimientos, innovaciones y prácticas.

15 El cual señala que los pueblos indígenas están facultados para controlar el acceso a sus conocimientos, innovaciones y prácticas, los que constituyen su patrimonio cultural.

16 Artículo 2, inciso 19, de la Constitución Política del Perú.

17 Artículo 11, Ley $N^{\circ} 27811$.
} 


\section{DEFINICIÓN DE PUEBLOS INDÍGENAS}

La Ley $\mathrm{N}^{\circ}$ 27811, incluye en su definición de Pueblos Indígenas a los pueblos en aislamiento voluntario o no contactados, así como a las comunidades campesinas y nativas. Si bien, en un momento inicial se consideró señalar por separado las definiciones de comunidades campesinas, nativas y demás pueblos indígenas a fin de distinguir los derechos atribuidos a cada pueblo indígena y los que corresponden a una comunidad individual, finalmente se consideró que el no reconocimiento legal no era razón suficiente para excluir a estos pueblos indígenas del régimen propuesto, por lo que el texto de la Ley hizo referencia a Pueblos Indígenas de forma general.

Se hace notar que, la propuesta inicial decidió no hacer referencia a las comunidades afroamericanas en base al fundamento del reducido número de grupos afrodescendientes en el país y su proceso de aculturación (INDECOPI, 2000, p. 17).

\section{ÁMBITO DE PROTECCIÓN}

Es importante señalar que este Régimen sui generis fue posible en tanto las disposiciones expresas de la entonces Ley Propiedad Industrial aprobada por Decreto Legislativo $N^{\circ} 823$; además, señalar que la Ley $\mathrm{N}^{\circ} 27811$ recoge en su Primera Disposición Complementaria el principio de la independencia de la legislación vigente en materia de propiedad intelectual con relación a las disposiciones de las Decisiones 345 y 486 de la CAN.

\section{OBJETIVOS}

Nótese que la creación del régimen de protección tuvo como objeto "no sólo contribuir a preservar y transferir sino también a desarrollar los conocimientos de los pueblos indígenas"18 señalándose la necesidad de un marco legal con reglas claras a fin de crear mecanismos que promuevan la inversión en la investigación y desarrollo de los conocimientos tradicionales.

\section{REGISTROS DE CONOCIMIENTOS COLECTIVOS}

La Ley señala al INDECOPI como la institución encargada del Registro Nacional Público y el Registro Confidencial de Conocimientos Colectivos dejando la organización de los Registros Locales a los mismos Pueblos Indígenas, de conformidad con sus usos y costumbres. Los registros son uno de los mayores logros de la Ley $\mathrm{N}^{\circ} 27811$, en tanto constituyen el referente de los

\footnotetext{
18 En el Documento de Trabajo N003-1999
} 
esfuerzos del INDECOPI en la capacitación que se brinda a las comunidades sobre procedimiento para registrar sus conocimientos tradicionales y donde además se les brinda información de la utilidad del registro para proteger conocimientos y evitar la biopiratería. Hasta agosto de 2018, el INDECOPI ha otorgado un total de 5257 Títulos de Registro de Conocimientos Colectivos de los Pueblos Indígenas vinculados a los recursos biológicos ${ }^{19}$.

El consentimiento informado previo del que hablaremos más adelante, es parte fundamental en el proceso de la elaboración de cualquier registro o base de datos de conocimientos tradicionales, el mismo que por su naturaleza debe tener lugar en el mismo seno de la comunidad, y requiere además de un procedimiento previamente pactado en concordancia con sus costumbres, leyes y derecho consuetudinario de los Pueblos Indígenas.

\section{CONSENTIMIENTO INFORMADO PREVIO}

Establecido y reconocido el derecho de los Pueblos Indígenas sobre sus conocimientos tradicionales, es consecuente que recaiga en ellos como poseedores, la potestad de determinar si desean compartir los mismos con un público más amplio, así como las formas y términos de la apertura de la información, o en su defecto la decisión de no compartirlos. Así, el Consentimiento Informado Previo (PIC por sus siglas en inglés) es la suma de los requisitos que permiten contar con la autorización otorgada por poseedores del conocimiento tradicional para el acceso y uso de uno o varios de sus conocimientos, en conformidad con sus normas reconocidas y para la realización de una determina actividad; evitando así su uso inadecuado o no autorizado y constituyendo una medida de protección para que los mismos no sean puestos en el dominio público sin su debida autorización.

La Ley $\mathrm{N}^{\circ} 27811$ no brinda mayores orientaciones sobre los pasos para obtener el consentimiento, no obstante de la propia definición se desprenden ciertos requisitos para su debido otorgamiento, los cuales esperan desde hace 16 años ser oficialmente interpretados en la deseada reglamentación de la Ley. Se hace resaltar que la Ley también confiere protección al Pueblo Indígena ante la divulgación no autorizada por parte de un tercero con acceso legítimo al conocimiento tradicional pero con deber de reserva.

Igualmente, se enfatiza que para el acceso a los conocimientos con fines científicos es necesario contar con el consentimiento informado previo del

\footnotetext{
Nota de Prensa. Balance Primer Semestre 2018. Dirección de Invenciones y Nuevas Tecnologías del INDECOPI. Recuperado de: http://repositorio.indecopi.gob.pe/bitstream/handle/11724/6355/NP\%20180813\%20Balance $\% 20$ Conocimientos $\% 20$ colectivos.pdf? sequence $=1 \&$ isAllowed $=y$
} 
Pueblo Indígena poseedor del conocimiento; mientras que para el acceso con fines comerciales es además necesario suscribir un contrato de licencia e inscribirlo en el registro que implemente para estos fines el INDECOPI.

\section{SEGUNDA PARTE: LOS DESAFÍOS}

\section{RÉGIMEN DE BENEFICIOS MONETARIOS Y NO MONETARIOS}

Considerando la complejidad de conocer o asignar un valor real a los conocimientos colectivos durante el proceso de negociación para su acceso, y haciendo la salvedad que igualmente existen beneficios no monetarios como por ejemplo, aquellos que se pueden dar en la fase de investigación referidos a la transferencia de tecnología, capacitaciones, publicaciones conjuntas, etcétera; grosso modo el régimen sui generis de protección prevé dos tipos de compensaciones económicas:

1) Acceso a conocimientos colectivos con fines comerciales que no están en el dominio público: Para el cual en las cláusulas del contrato de licencia se deberá contemplar:

Un pago inicial o alguna forma de compensación directa inmediata;

Un pago no menor del 5\% del valor de las ventas brutas antes de impuestos resultantes de la comercialización de los productos desarrollados a partir de los conocimientos colectivos materia del contrato destinado a los pueblos indígenas;

Un porcentaje no menor del 10\% del valor de las ventas brutas antes de impuestos resultantes de la comercialización de los productos desarrollados a partir de los conocimientos destinados al Fondo para el Desarrollo de los Pueblos Indígenas.

2) "Regularización" de acceso a conocimientos colectivos que estén en el dominio público:

Para los conocimientos que hayan sido publicados entre el 08 de agosto de 1982 y el 08 de agosto de 2002, se deberá destinar un porcentaje (no definido) del valor de las ventas brutas, antes de impuestos, resultantes de la comercialización de los productos desarrollados en base a dicho conocimiento colectivo, para el Fondo para el Desarrollo de los Pueblos Indígenas 
La propuesta inicial del INDECOPI contemplaba que estas compensaciones sean determinadas por la libre negociación de las partes, lo cual parecía más coherente en tanto un contrato, es por definición, el acuerdo de la libre voluntad de las partes. En relación a la fórmula utilizada en los articulados que contemplan compensaciones económicas, se acota que en el documento de trabajo $\mathrm{N}^{\circ} 010-2000$ se indica que "se ha optado por establecer como unidad de referencia el valor de las ventas brutas antes de impuestos, en lugar de otros posibles indicadores, como los beneficios netos o las ventas netas, debido a su mayor simplicidad y en analogía con la figura utilizada en el caso de las regalías por concepto de derechos de autor".

Se ha comentado que la suscripción de contratos de licencia permitiría analizar si los altos porcentajes son factibles o no, pero ciertamente 16 años después de la entrada en vigencia de la Ley, los contratos de licencia brillan por su ausencia. Una de las razones principales serían justamente las disposiciones que establecen un alto porcentaje mínimo que impiden la posibilidad de negociar contratos de licencia de forma más abierta, lo que podría estar ocasionando que las empresas potencialmente interesadas hayan asumido una posición de cautela frente a las disposiciones de la Ley $\mathrm{N}^{\circ}$ 27811. Una alternativa sería una modificación de los porcentajes que permitiría a partes tener mayor libertad al momento de fijar la compensación en función del acceso de conocimiento, pudiendo inclusive utilizarse el sistema de regalías escalonadas.

\section{CONTRATOS DE LICENCIA}

La Ley $\mathrm{N}^{\circ} 27811$ señala que los conocimientos colectivos forman parte del Patrimonio Cultural de los Pueblos Indígenas, de forma tal que los mismos son inalienables e imprescriptibles; es decir, no pueden ser cedidos, vendidos, transferidos; sólo pueden ser objeto de licencias de uso.

De acuerdo a lo consignado en la Ley, quienes estén interesados en acceder a los conocimientos para fines de aplicación comercial o industrial, deberán: contactar, consultar y, negociar con el o los pueblos indígenas - poseedores del conocimiento colectivo-, un contrato de licencia de uso, el cual deberá ser obligatoriamente escrito, en idioma nativo y castellano; y por un plazo renovable no menor de un año ni mayor de 3 años, donde se prevean las condiciones para una adecuada retribución por dicho acceso y se garantice una distribución equitativa de los beneficios derivados del mismo. Los contratos de licencia no confieren exclusividad sobre el conocimiento colectivo y su inscripción en el registro del INDECOPI es obligatoria. 
Nuestro Código Civil señala que un contrato es acuerdo de dos o más partes para crear, regular, modificar o extinguir una relación jurídica patrimonial ${ }^{20}$; por lo que se asume que las condiciones obligatorias impuestas por la Ley $\mathrm{N}^{\circ} 27811$ en relación a los requisitos de forma, duración del contrato y la obligatoriedad de su inscripción en el registro del INDECOPI, se concibieron en aras que el INDECOPI ejerza una suerte de rol supervisor de los requisitos mencionados en el contrato de licencia a fin de salvaguardar los intereses y derechos de los Pueblos Indígenas.

Lo cierto es que desde la publicación de la Ley, a la fecha de la redacción del presente artículo, no existe ningún contrato de licencia registrado en el INDECOPI. Sin embargo, consideramos importante señalar la experiencia de los acuerdos suscritos en el marco del Programa Internacional de Cooperación para la Biodiversidad (ICBG) en el año 1996, antes de la promulgación de la Ley $\mathrm{N}^{\circ}$ 27811. Durante esta experiencia se suscribieron un contrato de recolección de material biológico, un acuerdo de opción de licencia, acuerdos de subcontratos y un contrato de licencia de know-how entre la Organización Central de Comunidades Aguarunas del Alto Marañón (OCAAM), la Federación de Comunidades Nativas Aguarunas del Río Nieva (FECONARIN) y la Federación Aguaruna del Río Dominguiza (FAD), así como su organización representativa nacional, la Confederación de Nacionalidades Amazónicas del Perú (CONAP) y Searle Pharmaceuticals (subsidiaria farmacéutica de Monsanto Inc.) (Tobin y Taylor, 2009, p. 40).

Las negociaciones realizadas entre 1993 y 1996 se llevaron a cabo en el marco de las disposiciones del Convenio de Diversidad Biológica y concluyeron en los acuerdos antes mencionados. En setiembre de 1999, ante la intención de no extender la licencia de know-how, Searle decidió cancelar la licencia (Greene, Shane, 2006, p. 23).

\section{FONDO PARA EL DESARROLLO DE LOS PUEBLOS INDÍGENAS}

El Fondo para el Desarrollo de los Pueblos Indígenas, en adelante el Fondo, secreó bajo el razonamiento quesiendolos conocimientos tradicionales compartidos por los Pueblos Indígenas, consecuente era lógico que todos los pueblos indígenas gocen de los beneficios que resulten de su acceso y utilización por parte de terceros. El porcentaje fijado - al menos el 10\% del valor de las ventas brutas - se desarrolló en el sentido que sería imposible identificar en cada contrato de licencia a todos los Pueblos Indígenas que posean el conocimiento objeto de la licencia; por lo que resultaba justo reservar un porcentaje de los beneficios para aquellos Pueblos Indígenas

20 Artículo 1351 del Código Civil Peruano. 
que posean el conocimiento tradicional aunque no hayan participado en la negociación. De esta manera, se buscó lograr que todos los Pueblos Indígenas obtengan un beneficio, al menos indirecto, en tanto la naturaleza colectiva del conocimiento tradicional.

Si trazamos una línea de tiempo en relación a los esfuerzos para implementar el Fondo, podemos mencionar que en 2011 se designaron a los miembros de su Comité Administrador mediante la Resolución Ministerial № 185-2011-MC. Este Comité Administrador realizó dos sesiones donde se aprobaron propuestas de artículos sin llegar a aprobarse una propuesta de reglamento completa ${ }^{21}$. En razón a los cambios en la dirigencia de las organizaciones representativas ${ }^{22}$, la Resolución Ministerial № 155-2013MC actualizó a los representantes designados, situación que se repitió nuevamente con la reciente Resolución Ministerial $\mathrm{N}^{\circ}$ 155-2018-MC, la cual designó como miembros del Comité Administrador a representantes de las siguientes organizaciones: CNA, ONAMIAP, FENMUCARINAP, UNCA, AIDESEP y dos representantes del Viceministerio de Interculturalidad del Ministerio de Cultura.

A pesar de lo expuesto, los esfuerzos por implementar el Fondo han sido incipientes recayendo la responsabilidad tanto en las organizaciones representativas de los Pueblos Indígenas, como en el Ministerio de Cultura en su rol de ente rector en materia de Pueblos Indígenas u Originarios ${ }^{23}$.

\section{AUTORIDAD NACIONAL COMPETENTE}

Si la Ley $\mathrm{N}^{\circ}$ 27811, encarga los registros públicos y confidencial de conocimientos colectivos al INDECOPI y lo establece como Autoridad Nacional Competente Administrativa para resolver todo lo relativo a la protección de los conocimientos colectivos; se hace mención que la Ley $\mathrm{N}^{\circ} 27811$ también otorga un rol importante a la participación de la Ley al Ministerio de Cultura en el Fondo para el Desarrollo de los Pueblos Indígenas y en el Consejo Especializado en la protección de conocimientos indígenas ${ }^{24}$; sin mencionar la reciente precisión del Ministerio de Cultura como Ente Rector en materia de Pueblos Indígenas u Originarios ${ }^{25}$. A la luz de este contexto, y en consideración

$21 \quad$ Acta del 20 de setiembre de 2012 donde se acordó que el Reglamento Interno regía desde la firma de la mencionada acta pero el mismo constituiría parte de la redacción del Reglamento del Fondo a ser aprobado mediante Decreto Supremo; y Acta del 4 de octubre del 2012 donde se aprobó la inclusión de artículos en la propuesta de Reglamento del Fondo.

22 La cual determinó como miembros a la CONACAMI, CNA, AIDESEP, CONAP, CCP y dos representante del Viceministerio de Interculturalidad del Ministerio de Cultura.

23 Decreto Legislativo $\mathrm{N}^{\circ} 1360$, Decreto Legislativo que precisa las funciones exclusivas del Ministerio de Cultura.

24 Tampoco implementado a la actualidad.

25 Decreto Legislativo $\mathrm{N}^{\circ} 1360$, Decreto Legislativo que precisa las funciones exclusivas del Ministerio de Cultura. 
a las competencias de ambas instituciones en materia de conocimientos tradicionales, se hace necesario coordinar esfuerzos interinstitucionales a fines de implementar la Ley $\mathrm{N}^{\circ} 27811$, un primer paso se ha dado a través del reciente Convenio Específico de Cooperación Interinstucional en materia de conocimientos tradicionales suscrito por ambas instituciones con fecha 15 de agosto de 2018 y referido principalmente al desarrollo de documentos pedagógicos y eventos de difusión en la materia ${ }^{26}$.

\section{CONCLUSIONES}

Transcurridos 16 años desde la entrada en vigencia de la Ley $\mathrm{N}^{\circ}$ 27811 , sus principales y más notorios logros recaen en la constitución per se del régimen especial de protección, la destacada labor del INDECOPI en la implementación de los registros confidencial y público a través de la capacitación a las comunidades poseedoras de estos conocimientos tradicionales, y la obligatoriedad de la obtención del consentimiento informado previo. No obstante, y dado que los componentes de la Ley se conceptualizaron en una natural interrelación, la eficacia práctica y valor de los logros mencionados no alcanzará su potencial deseado en tanto no existan contratos de licencia de uso de conocimientos tradicionales o un rol más activo por parte de los mismos Pueblos Indígenas en la interposición de denuncias por uso indebido de sus conocimientos tradicionales ante el INDECOPI. Igualmente la incipiente implementación del Fondo para el Desarrollo Indígena deja entrever una falta de compromiso por parte de las organizaciones representativas de los pueblos indígenas y especialmente del propio Estado, en tanto el desarrollo de proyectos integrales de los Pueblos Indígenas constituye per se la concretización práctica de los objetivos de la Ley.

\section{REFERENCIAS}

\section{Constitución Política del Perú (1993).}

Comisión Permanente del Congreso de la República (2002). Diario de Los Debates. Comisión Permanente $13^{\underline{a}}$ G Sesión de la Comisión Permanente del Congreso de la República.

\footnotetext{
26 Los compromisos conjuntos del Convenio Específico de Cooperación son: a) Elaborar un documento pedagógico sobre la participación justa y equitativa en los beneficios derivados la utilización de conocimientos colectivos de los Pueblos Indígenas u Originarios; b) Elaborar un documento pedagógico sobre el consentimiento informado previo de los pueblos indígenas u Originarios ; c) Elaborar un documento base sobre los registros locales de conocimientos colectivos de los Pueblos Indígenas u Originarios y d) Coordinar, cuando sea pertinente, conferencias , seminarios, talleres, foros, charlas, mesas de difusión entre otras, con relación a la protección de los conocimientos colectivos de los pueblos indígenas u originarios.
} 
Congreso de la República (2002). Dictamen de la Comisión de Asuntos Indígenas y Afroperuanos del Congreso de la República recaído en el Proyecto de Ley $N^{\circ}$ 2754/2001-CR, Proyecto de Ley 2439/2001-CR, y Proyecto de Ley 3275/2001-CR.

INDECOPI (1999). Propuesta de Régimen de Protección de los Conocimientos Colectivos de los Pueblos Indígenas y Acceso a los Recursos Genéticos Documento de Trabajo $N^{\circ}$ 003-1999. Área de Estudios Económicos. Preparado por Armando Cáceres, Ana María Pacón, Víctor Revilla, Gonzalo Ruíz y Begoña Venero; con la colaboración de Héctor Agui, Beatriz Boza, Hugo Eyzaguirre, Javier Mori y Demetrio Rojas. Lima, Perú.

INDECOPI (2000). Documento de Trabajo $\mathrm{N}^{\circ}$ 010-2000. Área de Estudios Económicos del INDECOPI. Propuesta de régimen de protección de los conocimientos colectivos de los Pueblos Indígenas (y algunas reflexiones sobre la regulación del acceso a los recursos genéticos). Preparado por Juan Luiz Avendaño Cisneros, Ana María Pacón, Gonzalo Ruiz y Begoña Venero, con la colaboración de Sylvia Bazán, Armando Cáceres y Beatriz Boza. Lima, Perú.

Greene, Shane (2006). Pueblos Indígenas? La cultura como política y propiedad enla bioprospección farmacéutica. Revista Colombiana de Antropología. Bogotá, Colombia: Instituto Colombiano de Antropología e Historia - ICANH.

Ley $\mathrm{N}^{\circ}$ 27811, Ley que establece el Régimen de Protección de los Conocimientos Colectivos de los Pueblos Indígenas vinculados a los Recursos Biológicos (24 de julio 2002).

Organización Internacional del Trabajo (1989). Convenio Nº 169 de la Organización Internacional del Trabajo sobre pueblos indígenas y tribales.

Registro de Expedientes del Congreso de la República. Departamento de investigación y documentación parlamentaria. Ley $\mathrm{N}^{\circ}$ 27811, Ley que establece el Régimen de Protección de los Conocimientos Colectivos de los Pueblos Indígenas vinculados a los recursos biológicos. Recuperado de: http://www2.congreso.gob.pe/Sicr/TraDocEstProc/CLProLey2001.nsf

Ruiz, Manuel (2006). Laprotección jurídica de los conocimientos tradicionales: Algunos Avances Políticos y Normativos en América Latina. Lima: Unión Mundial para la Naturaleza/ Sociedad Peruana de Derecho Ambiental.

Tobin, Brendan (2001). Redefining Perspectives in the Search for Protection of Traditional Knowledge: A Case Study from Peru. Review of European Community \& International Environmental Law. Volumen 10. Issue 1. 
Tobin, Brendan and Taylor Emily (2009). Across the Great Divide: A case study of complementarity and conflict between customary law and TK protection legislation in Peru. Initiative for the prevention of Biopiracy. Year IV $N^{\circ}$ 11. Lima, Perú. Sociedad Peruana de Derecho Ambiental.

Tobin Bredan y Swiderska Krystyna (2001). En busca de un lenguaje común: Participación indígena en el desarrollo de un régimen sui generis para la protección del conocimiento tradicional en el Perú. London, Inglaterra: International Institute for Environmental and Development (EIID).

Fecha de recepción : $\quad 29$ de agosto de 2018 Fecha de aceptación : : 25 de octubre 2018 